\title{
CHERN-SIMONS AND STRING THEORY
}

\author{
KISHORE MARATHE
}

Communicated by Martin Schlichenmaier

\begin{abstract}
We discuss a special case of the gauge theory to string theory correspondence where exact results are available. We show how the Witten-ReshetikhinTuraev invariant in $\mathrm{SU}(n)$ Chern-Simons theory on $S^{3}$ is related via conifold transition to the all genus generating function of the topological string amplitudes on a Calabi-Yau manifold.
\end{abstract}

\section{Introduction}

Last year several of us (lucky mathematical physicists) attended the Bayrischzell workshop 2004 on noncommutativity and physics. One of the speakers told us that gauge theory, open string theory, closed string theory and noncommutative QFT are the same. Perhaps the heady alpine air had something to do with this euphoric statement. Nevertheless several bits and pieces of evidence supporting the existence of links between these theories has accumulated over the last few years. However, stealing a line from Cliff Taubes, I would like to say that at least from a mathematical point of view we would be lucky if in a few years we know what are the right questions to ask. This year we are celebrating the 25th anniversary of the marriage between Gauge Theory and the Geometry of Fiber Bundles from the sometime warring tribes of Physics and Mathematics. Marriage brokers were none other than Chern and Simons. The 1978 paper by Wu and Yang can be regarded as the announcement of this union. It has led to many wonderful offspring. The theories of Donaldson, Chern-Simons, Floer-Fukaya, Seiberg-Witten, and TQFT are just some of the more famous members of their extended family. Quantum Groups, CFT, Supersymmetry, String Theory and Gravity also have close ties with this family. In this talk we will discuss one particular relationship that has recently come to light. The qualitative aspects of Chern-Simons theory as string theory were investigated by Witten [6] almost ten years ago. Before recounting the main idea of this work we review the Feynman path integral method of quantization which is particularly suited for studying topological quantum field 
theories. For general background on gauge theory and geometric topology see, for example, [2-4].

\section{Quantum Observables}

A quantum field theory may be considered as an assignment of the quantum expectation $\langle\Phi\rangle_{\mu}$ to each gauge invariant function $\Phi: \mathcal{A}(M) \rightarrow \mathbb{C}$, where $\mathcal{A}(M)$ is the space of gauge potentials for a given gauge group $G$. $\Phi$ is called a quantum observable or simply an observable in quantum field theory. In the Feynman path integral approach to quantization the quantum expectation $\langle\Phi\rangle_{\mu}$ of an observable is given by the following expression

$$
\langle\Phi\rangle_{\mu}=\frac{\int_{\mathcal{A}(M)} \mathrm{e}^{-S_{\mu}(\omega)} \Phi(\omega) \mathcal{D} \mathcal{A}}{\int_{\mathcal{A}(M)} \mathrm{e}^{-S_{\mu}(\omega) \mathcal{D} \mathcal{A}}}
$$

where $\mathcal{D} \mathcal{A}$ is a suitably defined measure on $\mathcal{A}(M)$. It is customary to express the quantum expectation $\langle\Phi\rangle_{\mu}$ in terms of the partition function $Z_{\mu}$ defined by

$$
Z_{\mu}(\Phi):=\int_{\mathcal{A}(M)} \mathrm{e}^{-S_{\mu}(\omega)} \Phi(\omega) \mathcal{D} \mathcal{A} .
$$

Thus we can write

$$
\langle\Phi\rangle_{\mu}=\frac{Z_{\mu}(\Phi)}{Z_{\mu}(1)} .
$$

In the above equations we have written the quantum expectation as $\langle\Phi\rangle_{\mu}$ to indicate explicitly that, in fact, we have a one-parameter family of quantum expectations indexed by the coupling constant $\mu$ in the action. There are several examples of gauge invariant functions. For example, primary characteristic classes evaluated on suitable homology cycles give an important family of gauge invariant functions. The instanton number $k$ of $P(M, G)$ belongs to this family, as it corresponds to the second Chern class evaluated on the fundamental cycle of $M$ representing the fundamental class $[M]$. The pointwise norm $\left|F_{\omega}\right|_{x}$ of the gauge field at $x \in M$, the absolute value $|k|$ of the instanton number $k$ and the YangMills action $S_{\mu}$ are also gauge invariant functions. Another important example is the Wilson loop functional well known in the physics literature.

Wilson loop functional: Let $\rho$ denote a representation of $G$ on $V$. Let $\alpha \in$ $\Omega\left(M, x_{0}\right)$ denote a loop at $x_{0} \in M$. Let $\pi: P(M, G) \rightarrow M$ be the canonical 
projection and let $p \in \pi^{-1}\left(x_{0}\right)$. If $\omega$ is a connection on $P$, then the parallel translation along $\alpha$ maps the fiber $\pi^{-1}\left(x_{0}\right)$ into itself. Let $\hat{\alpha}_{\omega}: \pi^{-1}\left(x_{0}\right) \rightarrow$ $\pi^{-1}\left(x_{0}\right)$ denote this map. Since $G$ acts transitively on the fibers, there exists $g_{\omega} \in G$ such that $\hat{\alpha}_{\omega}(p)=p g_{\omega}$. Now define

$$
\mathcal{W}_{\rho, \alpha}(\omega):=\operatorname{Tr}\left[\rho\left(g_{\omega}\right)\right] \text { for all } \omega \in \mathcal{A} .
$$

We note that $g_{\omega}$ and hence $\rho\left(g_{\omega}\right)$, change by conjugation if, instead of $p$, we choose another point in the fiber $\pi^{-1}\left(x_{0}\right)$, but the trace remains unchanged. We call these $\mathcal{W}_{\rho, \alpha}$ the Wilson loop functionals associated to the representation $\rho$ and the loop $\alpha$. In the particular case when $\rho=\operatorname{Ad}$ the adjoint representation of $G$ on $\mathfrak{g}$, our constructions reduce to those considered in physics. If $L=\left(\kappa_{1}, \ldots, \kappa_{n}\right)$ is an oriented link with component knots $\kappa_{i}, 1 \leq i \leq n$ and if $\rho_{i}$ is a representation of the gauge group associated to $\kappa_{i}$, then we can define the quantum observable $\mathcal{W}_{\rho, L}$ associated to the pair $(L, \rho)$, where $\rho=\left(\rho_{1}, \ldots, \rho_{n}\right)$ by

$$
\mathcal{W}_{\rho, L}=\prod_{i=1}^{n} \mathcal{W}_{\rho_{i}, \kappa_{i}} .
$$

\section{Link Invariants}

In the 1980s, Jones discovered his polynomial invariant $V_{\kappa}(q)$, called the Jones polynomial, while studying Von Neumann algebras and gave its interpretation in terms of statistical mechanics. These new polynomial invariants have led to the proofs of most of the Tait conjectures. As with the earlier invariants, Jones' definition of his polynomial invariants is algebraic and combinatorial in nature and was based on representations of the braid groups and related Hecke algebras. The Jones polynomial $V_{\kappa}(t)$ of $\kappa$ is a Laurent polynomial in $t$ (polynomial in $t$ and $t^{-1}$ ) which is uniquely determined by a simple set of properties similar to the well known axioms for the Alexander-Conway polynomial. More generally, the Jones polynomial can be defined for any oriented link $L$ as a Laurent polynomial in $t^{1 / 2}$.

A geometrical interpretation of the Jones' polynomial invariant of links was provided by Witten by applying ideas from QFT to the Chern-Simons Lagrangian constructed from the Chern-Simons action

$$
\mathcal{A}_{C S}=\frac{k}{4 \pi} \int_{M} \operatorname{tr}\left(A \wedge d A+\frac{2}{3} A \wedge A \wedge A\right)
$$


where $A$ is the gauge potential of the $\mathrm{SU}(n)$ connection $\omega$. Chern-Simons action is not gauge invariant. Under a gauge transformation $g$ the action transforms as follows

$$
\mathcal{A}_{C S}\left(A^{g}\right)=\mathcal{A}_{C S}(A)+2 \pi k \mathcal{A}_{W Z}
$$

where $\mathcal{A}_{W Z}$ is the Wess-Zumino action functional. It can be shown that the Wess-Zumino functional is integer valued and hence, if the Chern-Simons coupling constant $k$ is taken to be an integer, then the partition function $Z$ defined by

$$
Z(\Phi):=\int_{\mathcal{A}(M)} \mathrm{e}^{-\mathrm{i} \mathcal{A}_{C S}(\omega)} \Phi(\omega) \mathcal{D} \mathcal{A}
$$

is gauge invariant.

We denote the Jones polynomial of $L$ simply by $V$. Recall that there are 3 standard ways to change a link diagram at a crossing point. The Jones polynomials of the corresponding links are denoted by $V_{+}, V_{-}$and $V_{0}$ respectively. To verify the defining relations for the Jones' polynomial of a link $L$ in $S^{3}$, Witten starts by considering the Wilson loop functionals for the associated links $L_{+}, L_{-}, L_{0}$. Witten obtains the following skein relation for the polynomial invariant $V$ of the link

$$
t^{n / 2} V_{+}-t^{-n / 2} V_{-}=\left(t^{1 / 2}-t^{-1 / 2}\right) V_{0}
$$

where we have put

$$
\langle\Phi\rangle=V(t), \quad \text { and } \quad t=\mathrm{e}^{2 \pi \mathrm{i} /(k+n)} .
$$

We note that the result makes essential use of the Verlinde fusion rules in $2 d$ conformal field theory.

For SU(2) Chern-Simons theory, equation (1) is the skein relation that defines a variant of the original Jones' polynomial. This variant also occurs in the work of Kirby and Melvin where the invariants are studied by using representation theory of certain Hopf algebras and the topology of framed links. It is not equivalent to the Jones polynomial. In an earlier work I had observed that under the transformation $\sqrt{t} \rightarrow-1 / \sqrt{t}$, it goes over into the equation which is the skein relation characterizing the Jones polynomial. The Jones polynomial belongs to a different family that corresponds to the negative values of the level. Note that the coefficients in the skein relation (1) are defined for positive values of the level $k$. To extend them to negative values of the level we must also note that the shift in $k$ by the dual Coxeter number would now change the level $-k$ to $-k-n$. If in equation (1) we now allow negative values of $n$ and take $t$ to be a formal variable, then the extended family includes both positive and negative levels. 
Let $V^{(n)}$ denote the Jones-Witten polynomial corresponding to the skein relation (1), (with $n \in \mathbb{Z}$ ) then the family of polynomials $\left\{V^{(n)}\right\}$ can be shown to be equivalent to the two variable HOMFLY polynomial $P(\alpha, z)$ which satisfies the following skein relation

$$
\alpha P_{+}-\alpha^{-1} P_{-}=z P_{0} .
$$

If we put $\alpha=t^{-1}$ and $z=\left(t^{1 / 2}-t^{-1 / 2}\right)$ in equation (2) we get the skein relation for the original Jones polynomial $V$. If we put $\alpha=1$ we get the skein relation for the Alexander-Conway polynomial.

To compare our results with those of Kirby and Melvin we note that they use $q$ to denote our $t$ and $t$ to denote its fourth root. They construct a modular Hopf algebra $U_{t}$ as a quotient of the Hopf algebra $U_{q}(\mathfrak{s l}(2, \mathbb{C}))$ which is the well known $q$ deformation of the universal enveloping algebra of the Lie algebra $\mathfrak{s l}(2, \mathbb{C})$. Jones polynomial and its extensions are obtained by studying the representations of the algebras $U_{t}$ and $U_{q}$.

\section{WRT Invariants}

If $Z_{k}(1)$ exists, it provides a numerical invariant of $M$. For example, for $M=S^{3}$ and $G=\mathrm{SU}(2)$, using the Chern-Simons action Witten obtains the following expression for this partition function as a function of the level $k$

$$
Z_{k}(1)=\sqrt{\frac{2}{k+2}} \sin \left(\frac{\pi}{k+2}\right) .
$$

This partition function provides a new family of invariants of $S^{3}$. Such a partition function can be defined for a more general class of three-manifolds and gauge groups. More precisely, let $G$ be a compact, simply connected, simple Lie group and let $k \in \mathbb{Z}$. Let $M$ be a two-framed closed, oriented three-manifold. We define the Witten invariant $\mathcal{T}_{G, k}(M)$ of the triple $(M, G, k)$ by

$$
\mathcal{T}_{G, k}(M):=Z(1):=\int_{\mathcal{A}(M)} \mathrm{e}^{-\mathrm{i} \mathcal{A}_{C S}} \mathcal{D} \mathcal{A}
$$

where $\mathcal{D} \mathcal{A}$ is a suitable measure on $\mathcal{A}(M)$. We note that a precise definition of such a measure is not available at this time and the definition is to be regarded as a formal expression. Indeed, one of the aims of TQFT is to make sense of such formal expressions. We define the normalized Witten invariant $\mathcal{W}_{G, k}(M)$ of a 
two-framed, closed, oriented three-manifold $M$ by

$$
\mathcal{W}_{G, k}(M):=\frac{\mathcal{T}_{G, k}(M)}{\mathcal{T}_{G, k}\left(S^{3}\right)} .
$$

If $G$ is a compact, simply connected, simple Lie group and $M, N$ are two twoframed, closed, oriented three-manifolds. Then we have the following results

$$
\begin{aligned}
\mathcal{T}_{G, k}\left(S^{2} \times S^{1}\right) & =1 \\
\mathcal{T}_{\mathrm{SU}(2), k}\left(S^{3}\right) & =\sqrt{\frac{2}{k+2}} \sin \left(\frac{\pi}{k+2}\right) \\
\mathcal{W}_{G, k}(M \# N) & =\mathcal{W}_{G, k}(M) \mathcal{W}_{G, k}(N) .
\end{aligned}
$$

In his work Kohno defined a family of invariants $\Phi_{k}(M)$ of a three-manifold $M$ by its Heegaard decomposition along a Riemann surface $\Sigma_{g}$ and representations of its mapping class group in the space of conformal blocks. The agreement of his results (up to normalization) with those of Witten may be regarded as strong evidence for the usefulness of the ideas from TQFT and CFT in low dimensional geometric topology. We remark that a mathematically precise definition of the Witten invariants via solutions of the Yang-Baxter equations and representations of the corresponding quantum groups was given by Reshetikhin and Turaev. For this reason, we now refer to them as Witten-Reshetikhin-Turaev or WRT invariants. The invariant is well defined only at roots of unity and perhaps near roots of unity if a perturbative expansion is possible. This situation occurs in the study of classical modular functions and Ramanujan's mock theta functions. Lawrence and Zagier [1] have obtained several different formulas for the WRT invariant $\mathcal{W}_{\mathrm{SU}(2), k}(M)$ of the Poincaré homology sphere $M=\Sigma(2,3,5)$. They show how the Witten invariant can be extended from integral $k$ to rational $k$ and give its relation to the mock theta function. In particular, they obtain the following fantastic formula, a la Ramanujan, for the Witten invariant of the Poincaré homology sphere

$$
\mathcal{W}=1+\sum_{n=1}^{\infty} x^{-n^{2}}(1+x)\left(1+x^{2}\right) \ldots\left(1+x^{n-1}\right)
$$

where $x=\mathrm{e}^{\pi \mathrm{i} /(k+2)}$. We note that the series on the right hand side of this formula terminates after $k+2$ terms $^{1}$.

\footnotetext{
${ }^{1}$ I would like to thank Don Zagier for bringing this work to my attention
} 


\section{Chern-Simons and String Theory}

As we remarked at the beginning of this talk the question "what is the relationship between gauge theory and string theory?" is not meaningful at this time. However, interesting special cases where such relationship can be established are emerging. For example, Witten has argued that Chern-Simons gauge theory on a threemanifold $M$ can be viewed as a string theory constructed by using a topological sigma model with target space $T^{*} M$. The perturbation theory of this string will coincide with Chern-Simons perturbation theory, in the form discussed by Axelrod and Singer. The coefficient of $k^{-r}$ in the perturbative expansion of $\mathrm{SU}(n)$ theory in powers of $1 / k$ comes from Feynman diagrams with $r$ loops. Witten shows how each diagram can be replaced by a Riemann surface $\Sigma$ of genus $g$ with $h$ holes (boundary components) with $g=(r-h+1) / 2$. Gauge theory would then give an invariant $\Gamma_{g, h}(M)$ for every topological type of $\Sigma$. Witten shows that this invariant would equal the corresponding string partition function $Z_{g, h}(M)$. We now give an example of gauge theory to string theory correspondence relating the non-perturbative WRT invariants in Chern-Simons theory with gauge group $\mathrm{SU}(n)$ and topological string amplitudes which generalize the $\mathrm{GW}$ (Gromov-Witten) invariants of Calabi-Yau three-folds. The passage from real three dimensional Chern-Simons theory to the ten-dimensional string theory and further onto the eleven-dimensional M-theory can be schematically represented by the following

$$
\begin{aligned}
3+3 & =6(\text { real symplectic } 6 \text {-manifold }) \\
& =6\left(\text { conifold in } \mathbb{C}^{4}\right) \\
& =6(\text { Calabi-Yau manifold }) \\
& =10-4 \text { (string compactification) } \\
& =(11-1)-4 \text { (M-theory). }
\end{aligned}
$$

We now discuss the significance of the various terms of the above equation array. The first line suggests that we consider open topological strings on the cotangent bundle $T^{*} S^{3}$ with Dirichlet boundary conditions on the zero section $S^{3}$. We can compute the open topological string amplitudes from the $\mathrm{SU}(n)$ ChernSimons theory. Conifold transition [5] has the effect of closing up the holes in open strings to give closed strings on the Calabi-Yau manifold obtained by the usual string compactification from ten dimensions. Thus we recover a topological gravity result starting from gauge theory. In fact, as we discussed earlier, Witten had anticipated such a gauge theory string theory correspondence almost ten 
years ago. Significance of the last line is based on the conjectured equivalence of M-theory compactified on $S^{1}$ to type IIA strings compactified on a Calabi-Yau threefold.

\section{Conifold Transition}

To understand the relation of the WRT invariant of $S^{3}$ for SU $(n)$ Chern-Simons theory with open and closed topological string amplitudes on "Calabi-Yau" manifolds we need to discuss the concept of conifold transition. From the geometrical point of view this corresponds to symplectic surgery in six dimensions. It replaces a vanishing Lagrangian three-sphere by a symplectic $S^{2}$. The starting point of the construction is the observation that $T^{*} S^{3}$ minus its zero section is symplectomorphic to the cone $z_{1}^{2}+z_{2}^{2}+z_{3}^{2}+z_{4}^{2}=0$ minus the origin in $\mathbb{C}^{4}$, where each manifold is taken with its standard symplectic structure. The complex singularity at the origin can be smoothed out by the manifold $M_{\tau}$ defined by $z_{1}^{2}+z_{2}^{2}+z_{3}^{2}+z_{4}^{2}=\tau$ producing a Lagrangian $S^{3}$ vanishing cycle. There are also two so called small resolutions $M^{ \pm}$of the singularity with exceptional set $\mathbb{C P}^{1}$.

They are defined by

$$
M^{ \pm}:=\left\{z \in \mathbb{C}^{4} \quad ; \quad \frac{z_{1}+\mathrm{i} z_{2}}{z_{3} \pm \mathrm{i} z_{4}}=-\frac{-z_{3} \mp \mathrm{i} z_{4}}{z_{1}-\mathrm{i} z_{2}}\right\}
$$

Note that $M_{0} \backslash\{0\}$ is symplectomorphic to each of $M^{ \pm} \backslash \mathbb{C P}^{1}$. Blowing up the exceptional set $\mathbb{C P}^{1} \subset M^{ \pm}$gives a resolution of the singularity which can be expressed as a fiber bundle $F$ over $\mathbb{C P}^{1}$. Going from the fiber bundle $T^{*} S^{3}$ over $S^{3}$ to the fiber bundle $F$ over $\mathbb{C P}^{1}$ is referred to in the physics literature as the conifold transition. We note that holomorphic automorphism of $\mathbb{C}^{4}$ given by $z_{4} \mapsto-z_{4}$ switches the two small resolutions $M^{ \pm}$and changes the orientation of $S^{3}$. Conifold transition can also be viewed as an application of mirror symmetry to Calabi-Yau manifolds with singularities. Such an interpretation requires the notion of symplectic Calabi-Yau manifolds and the corresponding enumerative geometry.

\section{WRT Invariants and Topological String Amplitudes}

To find the relation between the large $n$ limit of $\mathrm{SU}(n)$ Chern-Simons theory on $S^{3}$ to a special topological string amplitude on a Calabi-Yau manifold we begin by recalling the formula for the partition function (vacuum amplitude) of the theory 
$\mathcal{T}_{\mathrm{SU}(n), k}\left(S^{3}\right)$ or simply $\mathcal{T}$. Up to a phase, it is given by

$$
\mathcal{T}=\frac{1}{\sqrt{n(k+n)^{(n-1)}}} \prod_{j=1}^{n-1}\left[2 \sin \left(\frac{j \pi}{k+n}\right)\right]^{n-j} .
$$

Let us denote by $F_{(g, h)}$ the amplitude of an open topological string theory on $T^{*} S^{3}$ of a Riemann surface of genus $g$ with $h$ holes. Then the generating function for the free energy can be expressed as

$$
-\sum_{g=0}^{\infty} \sum_{h=1}^{\infty} \lambda^{2 g-2+h} n^{h} F_{(g, h)} .
$$

This can be compared directly with the result from Chern-Simons theory by expanding the $\log \mathcal{T}$ as a double power series in $\lambda$ and $n$.

Instead of that we use the conifold transition to get the topological amplitude for a closed string on a Calabi-Yau manifold. We want to obtain the large $n$ expansion of this amplitude in terms of parameters $\lambda$ and $\tau$ which are defined in terms of the Chern-Simons parameters by

$$
\lambda=\frac{2 \pi}{k+n}, \quad \tau=n \lambda=\frac{2 \pi n}{k+n} .
$$

The parameter $\lambda$ is the string coupling constant and $\tau$ is the 't Hooft coupling $n \lambda$ of the Chern-Simons theory. The parameter $\tau$ has the geometric interpretation as the Kähler modulus of a blown up $S^{2}$ in the string amplitude expansion. If $F_{g}(\tau)$ denotes the amplitude for a closed string at genus $g$ then we have

$$
F_{g}(\tau)=\sum_{h=1}^{\infty} \tau^{h} F_{(g, h)} .
$$

So summing over the holes amounts to filling them up to give the closed string amplitude.

The large $n$ expansion of $\mathcal{T}$ in terms of parameters $\lambda$ and $\tau$ is given by

$$
\mathcal{T}=\exp \left[-\sum_{g=0}^{\infty} \lambda^{2 g-2} F_{g}(\tau)\right]
$$

where $F_{g}$ defined in (12) can be interpreted on the string side as the contribution of closed genus $g$ Riemann surfaces. For $g>1$ the $F_{g}$ can be expressed in terms 
of the Euler characteristic $\chi_{g}$ and the Chern class $c_{g-1}$ of the Hodge bundle of the moduli space $\mathcal{M}_{g}$ of Riemann surfaces of genus $g$ as follows

$$
F_{g}=\int_{\mathcal{M}_{g}} c_{g-1}^{3}-\frac{\chi_{g}}{(2 g-3) !} \sum_{n=1}^{\infty} n^{2 g-3} \mathrm{e}^{-n(\tau)} .
$$

The integral appearing in the formula for $F_{g}$ can be evaluated explicitly to give

$$
\int_{\mathcal{M}_{g}} c_{g-1}^{3}=\frac{(-1)^{(g-1)}}{(2 \pi)^{(2 g-2)}} 2 \zeta(2 g-2) \chi_{g} .
$$

The Euler characteristic is given by the Harder-Zagier formula

$$
\chi_{g}=\frac{(-1)^{(g-1)}}{(2 g)(2 g-2)} B_{2 g}
$$

where $B_{2 g}$ is the $(2 g)$-th Bernoulli number. We omit the special formulas for the genus 0 and genus 1 cases. The formulas for $F_{g}$ for $g \geq 0$ coincide with those of the $g$-loop topological string amplitude on a suitable Calabi-Yau manifold. This result can be extended to show that the expectation value of the quantum observable defined by the Wilson loop in the Chern-Simons theory also has a similar interpretation in terms of a topological string amplitude. The change in geometry that leads to this calculation can be thought of as the result of coupling to gravity. Such a situation occurs in the quantization of Chern-Simons theory. Here the classical Lagrangian does not depend on the metric, however, coupling to the gravitational Chern-Simons term is necessary to make it TQFT. It is well known that every closed connected oriented three-manifold can be obtained by surgery on a framed link in $S^{3}$. Moreover, such a three-manifold is the boundary of a fourmanifold which is a four-ball with finitely many two-handles attached. It would be interesting to relate invariants of four-manifolds to the quantum invariants of three-manifolds. The use of Wilson loops reminds us of Ashtekar's formalism for gravity. Chern-Simons theory is also closely related to the spin network formulation of three-dimensional quantum gravity. Recently Kontsevich has proposed an extension of TQFT by using some old ideas of Grothendieck. Perhaps such a theory may be viewed as a time evolution of three-dimensional Chern-Simons states. Following a proposal of Ooguri, Crane and Yetter have found a new invariant of a compact oriented four-manifold $M$ by applying ideas from modular tensor category to the three-skeleton of $M$. This invariant has an analytic expression in terms of the level $k$ WRT invariant of the SU(2) Chern-Simons theory and 
the corresponding string coupling constant and two classical topological invariants of $M$, namely, the Hirzebruch signature $\sigma(M)$ and the Euler characteristic $\chi(M)$. We note that Temperley-Lieb algebras associated to link diagrams and the Kirillov-Reshetikhin approach to link invariants via representations of the quantum deformation of the universal enveloping algebra of $\mathfrak{s u}(2)$, play a crucial role in obtaining this invariant. It can be thought of as a local combinatorial description of $\sigma(M)$, in the spirit of Gelfand and Macpherson's interpretation of Pontryagin classes.

A possible extension of the correspondence discussed in this paper to the case when Wilson loop variables are inserted on the Chern-Simons side and the physical interpretation of the string amplitudes is under investigation.

\section{Acknowledgements}

This work was supported in part by a research fellowship of the Max Planck Gesellschaft at the Max Planck Institute for Mathematics in the Sciences, Leipzig. I would like to thank Prof. Dr. Eberhard Zeidler for his continued interest in my work.

\section{References}

[1] Lawrence R. and Zagier D., Modular Forms and Quantum Invariants of 3-Manifolds, Asian J. Math. 3 (1999) 93-108.

[2] Marathe K. and Martucci G., The Mathematical Foundations of Gauge Theories, North-Holland, Amsterdam, 1992.

[3] Marathe K., Martucci G. and Francaviglia M., Gauge Theory, Geometry and Topology, Seminario di Matematica dell'Università di Bari, 262 (1995) $1-90$.

[4] Marathe K., A Chapter in Physical Mathematics: Theory of Knots in the Sciences, Springer, Berlin, 2001, pp. 873-888.

[5] Smith I., Thomas R. and Yau S.-T., Symplectic Conifold Transitions, J. Diff. Geom. 62 (2002) 209-242.

[6] Witten E., Chern-Simons Gauge Theory as String Theory, Prog. Math. 133 (1995) 637-678. 
Kishore Marathe

Department of Mathematics

Brooklyn College, CUNY

Brooklyn, NY 11210, USA

E-mail address: kmarathe@brooklyn. cuny . edu 